\title{
Ultimi sviluppi critici sulla narrativa tabucchiana
}

\author{
Flavia Brizio-Skov
}

Il numero speciale di Spunti e Ricerche (Bruno Ferraro e Nicole Prunster, eds. Antonio Tabucchi: a Collection of Essays. Spunti e Ricerche 12 [1996-97]: 3225) costituisce la prima collezione in inglese di saggi critici sulle maggiori opere narrative di Antonio Tabucchi. L'antologia, attraverso i quindici studi in essa contenuti, offre una visione ampia, ma allo stesso tempo precisa, delle principali tematiche che popolano il complesso universo narrativo dello scrittore toscano. Il volume deve essere accolto con plauso per il fatto di essere stato messo in circolazione in un momento ideale: essendo ormai state tradotte in inglese quasi tutte le opere dello scrittore (pubblicate da New Directions), essendo cresciuta la fama internazionale di Tabucchi grazie ai numerosi premi italiani ed esteri, ed anche ai successi sia di pubblico che di critica dei suoi romanzi e del suo recentissimo lavoro, La testa perduta di Damasceno Monteiro. È opportuno quindi sottolineare l'ottimo lavoro fatto dai due curatori, Bruno Ferraro e Nicole Prunster, ai quali non solo si deve attribuire il 'tempismo' dell'uscita del volume, ma anche la brillante idea di uno studio ad ampio respiro che sarà di grande aiuto allo specialista $e$, in special modo, al lettore di lingua inglese che si avvicinerà per la prima volta alle opere di Tabucchi.

Il volume si apre con un saggio introduttivo di Bruno Ferraro ("Antonio Tabucchi's Actors, Characters and Ghosts") che passa in rassegna l'opus di Tabucchi, le tematiche e lo stile della sua narrativa, intercalando commenti critici a brani di interviste fatte allo scrittore, e conclude con accenni al contenuto dei saggi che seguono. Dopo l'articolo di Ferraro, la raccolta presenta quattro sezioni, intitolate rispettivamente: "Narrativa e società", "Tempo, ricordi e sogni", "Immagini e paesaggi", "Il viaggio e la "quest'", "Storia e letteratura: Sostiene Pereira".

Nella prima sezione ("Narrativa e societa"), Giorgio Bertone ("Notes for a Reconnaissance of Tabucchi's Works") esamina le tecniche narrative di "Lettera da Casablanca" (da Il gioco del rovescio). Partendo dallo studio dei tempi verbali, il critico fa notare come questo alternarsi modale renda il racconto simultaneamente presente e passato agli occhi del lettore. Questa mescolanza infrange le tradizionali categorie temporali, contraendo e raccor- 
ciando il tempo lungo un tessuto narrativo che diventa un collage di frammenti, di ricordi, di peregrinazioni, di oggetti, di vecchie foto, di immagini di film. Il tempo tabucchiano è per Bertone un tempo interiore di ricordi visti come desideri perduti, come desideri di altre storie, di altre vite che affiorano dal passato individuale dei protagonisti e che emergono dalle numerose possibilità che il caso ha loro offerto o negato. Le vite di molti personaggi tabucchiani sono tessute quindi intorno a un ricordo, un gesto, un oggetto, un dettaglio e, nonostante la realtà del mondo fenomenico in cui si attuano, appartengono all'immaginario e non al reale. I protagonisti di queste storie hanno un io diviso, frammentato, che si definisce nella pluralità, nella crisi, nell'incertezza, nel rimorso e non nell'unità. "Come nuvole o elaborati cirri di fumo di sigaretta che si dipanano nell'aria e poi spariscono", i "plot" di Tabucchi, secondo Bertone, sembrano svanire per poi ricuperare consistenza quando il "piccolo equivoco" riacquista tutto a un tratto la sua preminenza, la sua chiarezza e la sua centralità nella trama narrativa. Tabucchi non fa rivivere il passato, bensì "mortifica" il presente. Infatti, mentre i suoi protagonisti sono immersi nell 'oggi, l'autore trasforma continuamente con abile tecnica narrativa, questo presente in passato sotto gli occhi dei personaggi e dei lettori. Il punto di incontro tra presente e passato assomiglia però alla linea dell'orizzonte che, come la ricerca di Spino (Il filo dell' orizzonte), diventa la ricerca del punto dove infanzia, ricordi e esperienza si intersecano e si illuminano: un luogo irraggiungibile. L'inconscio, ovvero la coscienza dei personaggi tabucchiani è la vera anima della narrazione, l'ordine temporale e spaziale tradizionali lasciano il posto al caso e alla probabilità che governano questa narrativa.

Remo Bodei ("The Geometry of Equality: Antonio Tabucchi and the Civil Dimension") sviluppa invece l'aspetto esistenzialista della narrativa tabucchiana, osservando che, anche nei romanzi come Sostiene Pereira o Piazza d'Italia, nei quali si agita un innegabile spirito anarchico che simpatizza per coloro che si ribellano contro chi detiene il potere, la crisi di coscienza dei protagonisti trasforma il politico in uno dei tanti elementi comuni alla vita di ciascuna persona. Il politico è qualcosa di importante, ma sempre indelebilmente connesso a altri fattori, quali i ricordi, l'infanzia, il lato nascosto delle cose, il caso, l'equivoco, i morti, i fantasmi.

Nella seconda parte della raccolta, intitolata "Tempo, ricordi e sogni", Inge Lanslots ("Tabucchi's Waiting Rooms") studia l' "attesa', ovvero il tempo passato dai protagonisti tabucchiani ad aspettare gli eventi e la tensione creata da questa attesa, concettualizzando la struttura temporale entro la quale questa si sviluppa. Basandosi sullo schema di Wittgenstein sulle categorie dell'attesa, la studiosa mette in luce due aspetti del 'tempo interstiziale': il tempo funzionale, di solito notturno, concepito come locus propizio alle rivelazioni, e la 'pausa' creata dalla 'quest' del protagonista nel suo cammino verso l'incontro risolutivo, nel caso di Requiem, ad esempio, quello con 
l"ospite'. Poiché i personaggi che si devono incontrare sono introvabili o morti, l'aspettativa per l'evento futuro non è altro che una 'resurrezione' del passato. Il 'non-tempo' e la 'non-fine' sono sinonimi che additano la natura passiva dell'attesa nella narrativa tabucchiana, il cui ritmo è caratterizzato dal 'movimento al rallentatore', da un dinamismo passivo che crea uno spazio ideale nel quale prospera un narrare senza fine.

Laura Lepschy invece ("The Role of Memory in Antonio Tabucchi's Piccoli equivoci senza importanza") nota che quello tabucchiano è sovente una sorta di tempo circolare nel quale si passa dal presente al passato per tornare poi al presente e così via, ma nel quale i ricordi hanno sempre la supremazia. La studiosa fornisce vari esempi di come la memoria operi nei racconti, come crei un'atmosfera di ambiguità e di mistero attorno ai personaggi.

Con grande accuratezza critica Nives Trentini ("Towards a Study of Dream in Antonio Tabucchi") compie uno studio molto approfondito del sogno in Tabucchi, affermando che persino nei romanzi più ancorati al reale quali Piazza d'Italia e Sostiene Pereira, il sogno è sempre un elemento determinante per la caratterizzazione dei personaggi, essendo un luogo dove reale e irreale, veglia e sonno, vero e falso si incontrano e si mescolano. In Piazza $D^{\prime}$ Italia il sogno, pur non avendo una posizione predominante come in altri romanzi, si manifesta in due aspetti: come proiezione del desiderio o come incubo, premonizione o anticipazione del futuro. Attraverso un'analisi dettagliata di Piazza la studiosa conclude che il sogno a volte diventa riproduzione di un desiderio che si attua nella scrittura come nel caso delle varie 'storie nella storia'. La Trentini passa poi all'esame di Requiem, da lei considerato un macro-sogno contenente diversi micro-sogni, ovvero i monologhi del protagonista con i personaggi-fantasma; piccoli sogni questi che però avvengono all'interno di uno scenario che sembra avere tutte le caratteristiche del reale. Tra il realismo delle descrizioni e l'irrealtà dei personaggi il sogno in Requiem finisce perciò coll'arricchire la realtà, giacché analizza, corregge, spiega una parte del vissuto rimasta bloccata, incompleta. Il sogno finisce con l'implementare la memoria e riordinare attraverso connessioni apparentemente illogiche le sofferenze dell'esistenza, rimarginando le disarmonie del vissuto.

Nel terzo segmento, "Immagini e paesaggi", Tiziana Arvigo ("From Notturno indiano to Il filo dell' orizzonte: 'landscape of absence' and 'landscape of disappearance" ") esamina lo "sguardo" nella narrativa tabucchiana, trovando somiglianze tra questo sguardo e quello de Il libro dell'inquietudine. La studiosa sostiene che lo sguardo tabucchiano è capace di dirigersi verso "una vita esterna e reale che si svolge estranea a lui [...] e una vita interiore e inventata", creando così due paesaggi che si intersecano e si confondono, come avviene anche a Bernardo Soares, l'eteronimo di Pessoa. Con la differenza che in Tabucchi il paesaggio interiore non nasce dall'immaginazione 
dei personaggi come in Pessoa, ma dal passato, dal desiderio un po' presuntuoso di trovare "il nesso che unisce le cose". Lo scenario interiore ed esteriore del protagonista di Notturno Indiano, ad esempio, caratterizzato dall'invisibilità del paesaggio geografico. L'India è un paese fantasmagorico, nascosto, notturno, sembra emergere da un sogno, è una sorta di paese misterioso, che fa da controcanto al mistero della ricerca del protagonista.

Remo Ceserani ("The Art of Fixing Shadows and Writing with Light"), dopo una lunga carrellata sul significato della fotografia che spazia da Fredric Jameson a Walter Benjamin, da Mario Praz a Françoise Gilot, una volta constatata l'importanza che la fotografia ha in quasi tutti i romanzi tabucchiani. si concentra sulla funzione che essa assume ne Il filo dell' orizzonte. Questo è per Ceserani un romanzo con fotografie e scritte da decifrare, geroglifici da decodificare. I personaggi stessi interpretano film, fotografie, affreschi, scritte su monumenti, misteriosi messaggi e pagine di libri aperti per caso. Il testo è una sorta di 'decifrazione' continua. Nel romanzo la fotografia fa sì che la ricerca dell'identità del ragazzo morto venga sostituita dalla ricerca del significato della vita del protagonista. Spino "legge" nella foto del giovane ucciso quesiti che appartengono al dramma della sua identità divisa e in ultima analisi al dramma della condizione umana in generale; la foto non offre nessuna soluzione, bensì offre un doppio, un altro spazio nel quale il protagonista penetra grazie all'obiettivo. Tuttavia, il mistero, racchiuso negli occhi del ragazzo fotografato da bambino, rimane insondabile; è impossibile penetrare la sua realtà, così come alla fine del romanzo il sorriso che Spino lancia, prima di avanzare nel buio, resta impenetrabile al lettore.

Giovanni Palmieri (“Antonio Tabucchi’s Iconic Temptations”) afferma che la pittura e in generale la natura visiva delle immagini verbali hanno un'importanza decisiva nella narrativa tabucchiana. Notturno indiano è sospeso tra la tenebra del mistero dell'esistenza e la luce della ragione che vuole penetrare quelle tenebre senza però riuscirvi. È l'opposizione tra immagini di luce e d'ombra che porta avanti il significato e l'atmosfera del romanzo. I numerosi riferimenti pittorici che abbondano nella narrativa tabucchiana hanno funzioni diverse. Il riferimento pittorico a Mantegna serve a stabilire il punto di vista del protagonista, a spiegare qualcosa che senza l'aiuto della connotazione iconica sarebbe altrimenti difficile da comunicare. "Las Meninas" di Velásquez invece esemplifica un modo particolare di guardare il mondo, osservandolo dal rovescio. Il dettaglio, che il Copista de "Le tentazioni di Sant'Antonio" di Bosch riproduce in dimensioni ingigantite, acquista un significato del tutto indipendente dal contesto dal quale è tratto, così come la foto mostrata dalla giomalista al protagonista di Notturno Indiano, nella sua qualità di 'morceau choisi', diventa anch'essa un dettaglio staccato dal tutto. Il frammento si trasforma in una sorta di 'mise en abyme' della narrazione stessa; infatti il lettore non può mai essere sicuro di averne afferrato il significato, poiché questo apre il testo a molteplici interpretazioni. 
Nella penultima parte del libro, intitolata "Il viaggio e la "quest", Anna Botta (“Antonio Tabucchi”s Requiem: Mourning Modernism") con la solita eleganza che contraddistingue i suoi saggi, sostiene, rifacendosi a Freud, che il protagonista di Requiem nel corso del suo sogno elabora il lutto e porta alla superficic il senso di perdita, sino a liberare l'inconscio dal dolore e a raggiungere in questo modo una sorta di tranquillità. L'io-protagonista all 'inizio di questa rivisitazione si trova in uno stato allucinatorio, psicotico, giacché ogni singolo ricordo o evento passato deve essere rivissuto da lui come fosse presente. Costui, per potersi liberare dal dolore e allo stesso tempo dal desiderio della presenza dei morti, deve incontrare costoro come fossero vivi, rivolgendogli vecchie domande e frequentando gli stessi luoghi di un tempo. Il lutto si elabora, secondo la Botta (e secondo Freud e Melanie Klein) riincorporando nell'ego gli oggetti perduti del desiderio e ri-internalizzando gli 'oggetti positivi' nel proprio inconscio. Dopo che il protagonista ha incontrato i vari personaggi e il padre, costui si sente sollevato: la sua orazione ha portato tranquillità, non solo ai morti, ma anche a lui stesso. Questo si compie grazie a dei processi di 'introiezione', a una sorta di 'digestione dei morti' che si attua attraverso numerosi piatti tipici della cucina portoghese: il cibo consumato dai personaggi diventa il viaticum, il veicolo di scambio tra vivi e morti. Inoltre, il processo di liberazione dal lutto non avviene tramite le lacrime, bensì tramite una sudorazione insolita e incontenibile. La studiosa conclude affermando che l'elaborazione del lutto in Requiem include sia Freud che il modernismo. Partendo dal pensiero di Vattimo sul postmoderno, la Botta poi traccia un intricato parallelismo nel quale risulta che il Copista sta a Bosch come l'io-protagonista sta a Freud, come Tabucchi sta a Pessoa. In quest'ottica la letteratura diventa una forma di terapia grazie alla quale Tabucchi riesce a offrire il suo viaticum postmoderno a Pessoa, al Portogallo e ai suoi angeli neri.

Luigi Surdich ("The Constant Search for Oneself") trova che Notturno indiano, Il filo dell'orizzonte e Requiem formano una sorta di trilogia basata sul tema della ricerca dell'identità e a volte della morte. La 'quest' tabucchiana pero non segue i modelli letterari convenzionali, non porta alla conoscenza, ma alla ri-conoscenza, e l'avventura si trasforma in una sorta di disavventura, essendo i protagonisti vittime di eventi incontrollabili. Questa ricerca conduce i personaggi e il lettore a confrontarsi con ciò che è enigmatico e a ponderare la relazione tra enigma e verita. In questi tre romanzi l'io narrante fa congetture e supposizioni, vivendo in bilico tra il dubbio e l'ipotesi. Le coincidenze della vita dei personaggi sono sorprendenti e inquietanti, quello che a tutta prima sembra decifrabile si rivela poi ingannevole, in questo modo i nessi tra cose e eventi rimangono inspiegabili, condannando i protagonisti all'instabilità e alla non-identità. L'immagine del filo dell'orizzonte che si sposta con chi guarda è il correlativo oggettivo di tutti e tre questi romanzi, in questa linea mobile viene esemplificata non tanto la mancanza di 
un punto di arrivo, ma il fatto che questo punto è un obiettivo perenne, irraggiungibile e inconoscibile.

Nell'ultima sezione, "Storia e letteratura: Sostiene Pereira", Manuela Bertone ("Paths to Testimony in Sostiene Pereira") fa uno splendido studio di Sostiene Pereira, dipanando un discorso critico che parte dal sottotitolo: "Una testimonianza". Nella sua testimonianza Pereira propone una versione personale dei fatti che potrebbe essere confutata da quella di qualche altro testimone, se esistesse. Costui è infatti insicuro, titubante su una quantità di cose, giacché afferma che non sa, non vuole parlare di questo, non ricorda, preferisce passare ad altro, ecc. Tuttavia, il depositario della testimonianza a sua volta sembra aderire il più possibile alla versione del vecchio giornalista: non fa domande, non solleva obiezioni, non confuta, ecc. Questo misterioso interlocutore che "sostiene" quello che Pereira dice sembrerebbe non far parte dell'ambiente giudiziario, la testimonianza del giornalista parrebbe piuttosto offerta ad un analista, il quale la registra nella maniera più distaccata possibile e, per far ciò, ricorre a quel "sostiene" che garantisce l'integrità degli eventi e ne impedisce la contaminazione. È grazie a costui che la storia presentata da Pereira diventa una 'testimonianza', infatti è lui che si assume la responsabilità di registrarla e di trasmetterla, instaurando una specie di circolarità fra chi fa la confessione e chi la riceve. La Bertone definisce l'operazione di Tabucchi "etica", nel senso che lo scrittore ha adottato i valori della "letteratura della testimonianza" in opposizione a quella dello "svago". La letteratura che diventa "arte dell'ascoltare per raccontare" è associata nel romanzo alla responsabilità che la letteratura ha di dire e trasmettere la verità; verità che per Tabucchi vuol dire ricercare la connessione tra le cose, ponderare l'enigma dell'esistenza, gli strani casi della vita e allo stesso tempo non avere reticenze di fronte agli aspetti orrendi della Storia e del nostro vivere. Trasmettere la verita equivale a non manipolare $i$ fatti in modo da dipingere una realtà che appaia assoluta. In ultima analisi la storia di Pereira narra di una memoria involontaria, passiva e silenziosa che diventa positiva, dinamica e attiva; una memoria che smette di proiettarsi nel passato e si proietta invece nel futuro; una memoria che, nel denunciare l'assassinio di Monteiro, "materializza" la memoria della Signora Delgado e di tutte le voci degli intellettuali-letterati dissidenti contenute nel libro, da Thomas Mann a Lorca, da Mauriac a Bernanos.

Monica Jansen ("What about Pereira? Can He Be Trusted?") con agilità critica da ginnasta olimpionico e una batteria di idee da spettacolo pirotecnico ha scritto un saggio ammirevole, ben equilibrato tra scientificità e creatività. Immaginando che il misterioso personaggio Pereira di T.S. Eliot abbia bussato un giorno alla porta di Tabucchi in cerca di un autore, e che tale personaggio abbia poi fatto venire in mente allo scrittore toscano un giornalista portoghese che era stato costretto all'esilio parigino per aver pubblicato un articolo contro il regime salazarista, la Jansen sostiene che Pereira ha trovato 
finalmente qualcuno dal quale farsi ascoltare e al quale confessarsi, giacché Tabucchi ha prestato la sua immaginazione di scrittore al personaggio. In questa luce il "sostiene Pereira" del romanzo sancisce una sorta di contratto tra autore e personaggio, per mezzo del quale si ottengono diversi risultati: il 'refrain' diventa un modo per tenere l'autore al di fuori della confessione e diventa un filtro tra l'onniscienza del narratore e la personalità del protagonista. Insomma Tabucchi diviene il 'recorder' della testimonianza, di 'una storia indiretta', narrata in maniera da impedirgli qualsiasi interferenza. La studiosa fa poi un'analisi dettagliata delle differenze tra romanzo e versione cinematografica di Sostiene Pereira, sottolineando aspetti che vanno dall'uso del 'refrain' alla rappresentazione della dittatura, dalla trasposizione di eventi all'uso della voce fuori campo, ecc. Il tema centrale del romanzo è per la Jansen la trasformazione di Pereira da uomo che vive nel passato a persona proiettata nel futuro, una maturazione esistenziale che lo porta a cambiare il suo modo di agire sociale e politico, cosa che avviene anche nel film, ma con enfasi diversa. Passando poi al discorso della confederazione delle anime, la Jansen paragona il senso di colpa di Pereira all'ansia del protagonista di Requiem, notando con acume che però a quest'ultimo, invece dei 'médicinsphilosophes', vengono in aiuto Freud, Pessoa con i suoi eteronimi e i fantasmi. A Pereira d'altro canto basta il Dottor Cardoso e la sua teoria sulla confederazione delle anime per aiutarlo a risolvere la scissione tra arte e vita. Per lui le due cose, incompatibili all'inizio della sua storia, cessano, dopo l'assassinio di Monteiro, di esistere antagonisticamente e cominciano a coesistere in una costellazione organica. La comunione tra arte e vita, letteratura e politica viene raggiunta anche grazie alle discussioni letterarie, così numerose nel romanzo. Attraverso gli esempi di Bernanos, Mauriac, Maritain, Ribeiro e altri Pereira comprende che la vita e l'arte diventano politica se il contesto storico in cui interagiscono non è una democrazia, ma una dittatura.

Tralascio di parlare del mio articolo ("Sostiene Pereira: The Crisis of the Intellectual between History and Literature") per ovvie ragioni; tuttavia vorrei dire che esso offre un'interpretazione del personaggio Pereira ancora diversa da quelle precedenti, inserendosi a meta strada tra il discorso della Bertone e quello della Jansen. La mia analisi si concentra soprattutto sulla crisi di Pereira e dimostra come la perdita di certezze dell'intellettuale si situi nello scontro tra Storia e Letteratura, tra realtà e cultura. Il volume si conclude con una breve nota di André Sempoux ("A Note on the Phrase "sostiene Pereira"') sui possibili significati della frase ripetuta ossessivamente da Pereira, con una bibliografia delle opere di Tabucchi pubblicate in inglese, seguita da una bibliografia critica di saggi inglesi e italiani, e infine con una breve nota sui critici e i curatori del libro.

Sarebbe difficile trovare delle pecche al volume, essendo il livello critico di tutti quanti i saggi molto elevato. Forse, volendo proprio cavillare, dovrei confessare che da studiosa di Tabucchi avrei desiderato vedere rappresentati 
nella raccolta Piccolo Naviglio, Sogni di Sogni, Gli ultimi tre giorni di Pessoa e altre opere meno conosciute dello scrittore, anche se mi rendo conto che un primo studio critico in inglese dovrebbe, come infatti fa, occuparsi delle opere e delle tematiche fondamentali di uno scrittore. Infine, vorrei aggiungere che il libro non sarebbe così apprezzabile se non fosse per la splendida resa in inglese dei saggi tradotti, il cui merito va all'accurato e difficile lavoro fatto dai traduttori (David Fairservice, Walter Musolino, John Gatt-Rutter, Gerardo Papalia, Antonio Pagliaro, Mirna Cicioni, Felix Siddell, Isabel Moutinho), al quale si accompagna un editing meticoloso. Infatti non capita spesso di avere tra le mani un testo così limpidamente immune da errori.

University of Tennessee, Knoxville 